\title{
Vehicle plate localization and extraction based on hough transform and bilinear operations
}

\author{
Masar Abed Uthaib ${ }^{1}$, Muayad Sadik Croock ${ }^{2}$ \\ ${ }^{1}$ Iraqi Commission for Computers and Informatics (ICCI), Informatics Institute for Postgraduate Studies, Iraq \\ ${ }^{2}$ Computer Engineering Department, University of Technology, Iraq
}

\begin{tabular}{l} 
Article Info \\
\hline Article history: \\
Received Feb 7, 2020 \\
Revised Apr 11, 2020 \\
Accepted Apr 25, 2020 \\
\hline
\end{tabular}

Keywords:

Hough transform Hough transform Image processing Sobel and bilinear operations morphological operation Vehicle plate localization

\begin{abstract}
In general, the extraction of the vehicle plate is a previous step of plate recognition, and it actively studied for several decades. Plate localization is used in various security and traffic applications. In this paper, the proposed method is efficient to localize a plate for the multinational countries. The proposed method consists of three levels. The first level is the preprocessing that contains several steps. The digital camera capture images have been taken about twenty meters from the car with zooming two to three meters. Images are resampled using the zooming technique (bilinear interpolation) that makes the dimension of image (1024 x 768) pixels. The resampled images are resized to (940x 730). These images are converted to grayscale (green channel), and the bilateral filter is applied for removing noise. The second level is plate detection that adopts morphological operations, image subtraction, and vertical edge detection (Sobel). At the last Connected component analysis and Hough transform are used. The third level is the Deskew plate that notifies the plate may skew so that Hough transform is used to detect the largest line segment. Then, the images are rotated using bilinear interpolation. About 860 images are tested for different countries (Iraq, Belarus, Armenia, Hungary), and the accuracy is $98.99 \%$ for extraction of the plate and $100 \%$ for the Deskew plate. Thus, the proposed system shows high efficiency in achievement.
\end{abstract}

Copyright $(2020$ Institute of Advanced Engineering and Science. All rights reserved.

\section{Corresponding Author:}

Masar Abed Uthaib,

Department of computer science,

Iraqi Commission for Computers and Informatics (ICCI),

Bagdad, Iraq.

Email: Masar.uthaib2018@gmail.com

\section{INTRODUCTION}

The human population increase, and the vehicle control takes considerable space. The extraction of a license plate is very significant in the monitoring of borders, intelligent transportation systems, and controlling of traffic flow. The security enforcement makes the use of car detection is essential, in case of detection of stolen cars, terrorist activities, and parking violations. It is a technology uses the image processing to recognize vehicles by their car plates. License plate detection approaches aim to discover rectangular regions in the image where the plates found. License plate recognition accuracy relies on the License plate detection efficiency. Several drawbacks effect on the localization of license plate, including noise, weather conditions, places of the plate in the car, non-prorate camera, complex of the scene, unwanted characters, the effectiveness of the plate, backgrounds of plates[1].

Several techniques have been developed for the license plate localization from the vehicle image in recent years. In [2], authors proposed a way to extract plate; they used Sliding Concentric Window for division the interested region and morphological( dilation and erosion); the accuracy of the system was 86.5 $\%$. In [3], the haze removal technique was employed to enhance the images. Then the improved image 
converted to grayscale, with the Wiener filter for removing the noise and then binaries the de-noising image were used to achieve connected component labeling. This is to detect the connected-objects in the image and use the aspect ratio to identify the plate with an accuracy of localization about 93.78\%.In [4], they used the CIE-XYZ technique for the detection of color and the scale-space channel for detection of the plate; the extraction accuracy was $94.23 \%$. In [5], they proposed method in any case, the car plate color, and size. The algorithm detected the back-light zone of the car to get rid of the complicated background where the plate is found. It calculated map of heuristic energy in the concerned region together with the dense edge part by using the process of the unique histogram. The accuracy was $90.4 \%$.

In [6], authors propped algorithm to detect plates with yellow and white background, where they use discrete wavelet transform for larger images, extraction accuracy was 97.8\%.In [7], the Sliding Window was adopted as well as a histogram of the oriented gradient, and Support Vector Machines to raise the detection scale. The accuracy of the system was $96 \%$. The authors of [8] converted the color image to gray to be used in vertical Sobel edge detection. Morphological operations were used to strengthen the edge and delete the weakest. The holes in the morphological is solved using image-filled. The binary mask was used to isolate plate regions from others after that connected component analysis applied to find the most extensive area, which was the license plate. The extraction accuracy was about 82\%. In [9], a hierarchical algorithm was used. The authors proposed cascade classifiers that consisted of training and detection phases, local binary pattern features, the method depends on an edge, also a way that rely on color, and plan of contrast. Its accuracy was $98.45 \%$. In [10], license plate cognizance was presented by Ocular Character Perception. The authors proposed conversion RGB image to grayscale, horizontal and vertical edge projection on the gray image, then low pass filter from which Passing histograms. Finally, the interest's region was segmented and extracted. The authors of [11] proposed license plate extraction for Iraqi plates by two methods: the morphological operations and vertical Sobel. In the second method, the image resized to half by using (image pyramid command) in Matlab. The resized image converted to grayscale. Sobel edge detection for extraction edges in the picture. Morphological dilation to make the objects in image thick. Median filter used to remove noise Morphological (dilation -erosion -fill holes) operations applied to the filtered-image. The accuracy of first and second was $98.33 \%$ and $81.66 \%$, respectively.

This paper suggests an effective system for license plate extraction from the car-images by using image processing techniques: morphological operations (Opening, Closing, Dilation, and Erosion), image subtraction, vertical Sobel operator for edge detection, and the connected component analysis. Firstly, the original image is preprocessed by converting it to gray-level ((green channel)) and then the bilateral filter and adaptive histogram equalization used. The proposed system is efficient in extraction with an accuracy of 98.99\%. It also shows high-performance efficiency in comparison with other methods presented in literature studies due to the use of the Deskew image. The proposed method obtains $100 \%$ of the rotating plates accuracy. Figure 1 shows the workflow of the suggested algorithm.

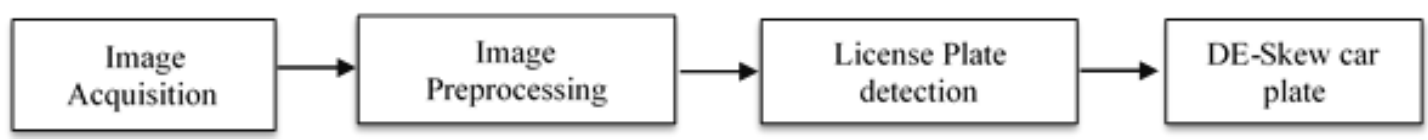

Figure 1. Proposed algorithm workflow

\section{THE PROPOSED METHOD}

The captured images are collected over different circumstances and various backgrounds. The proposed method is considered the required modification of the presented algorithm of [12]. Figure 2 displayed the flow chart of the suggested method. The major steps of the proposed system are summarized:

a) The captured images are resampled to $1024 \times 768$ using bilinear interpolation to decrease computation and preserve the image's quality.

b) For decreasing the number of pixels, images are resized to the size of $940 \times 730$ pixels.

c) For gray conversion, we use the green channel only as it satisfies the required efficiency with low complexity.

d) The bilateral-filter is applied to grey images for smoothing and removing noise. The histogram is used to know the number of pixels for each gray-scale-tonal.

e) After that, histogram equalization is adopted to equalize tonal variation.

f) Morphological is opened on adaptive-histogram and image subtraction.

g) Sobel edge detection and morphological dilation are applied to the subtracted images.

h) Morphological-open is added before applying fill-Holes. 
i) The other added operations are removing pixels less than 200 pixels after that morphological open and erosion, removing all objects except the six-biggest objects, analyzing the connected component to know the number of connected objects, extracting the license plate, Deskewing car plate using Hough transform for line detection and bilinear interpolation to rotate the plate.

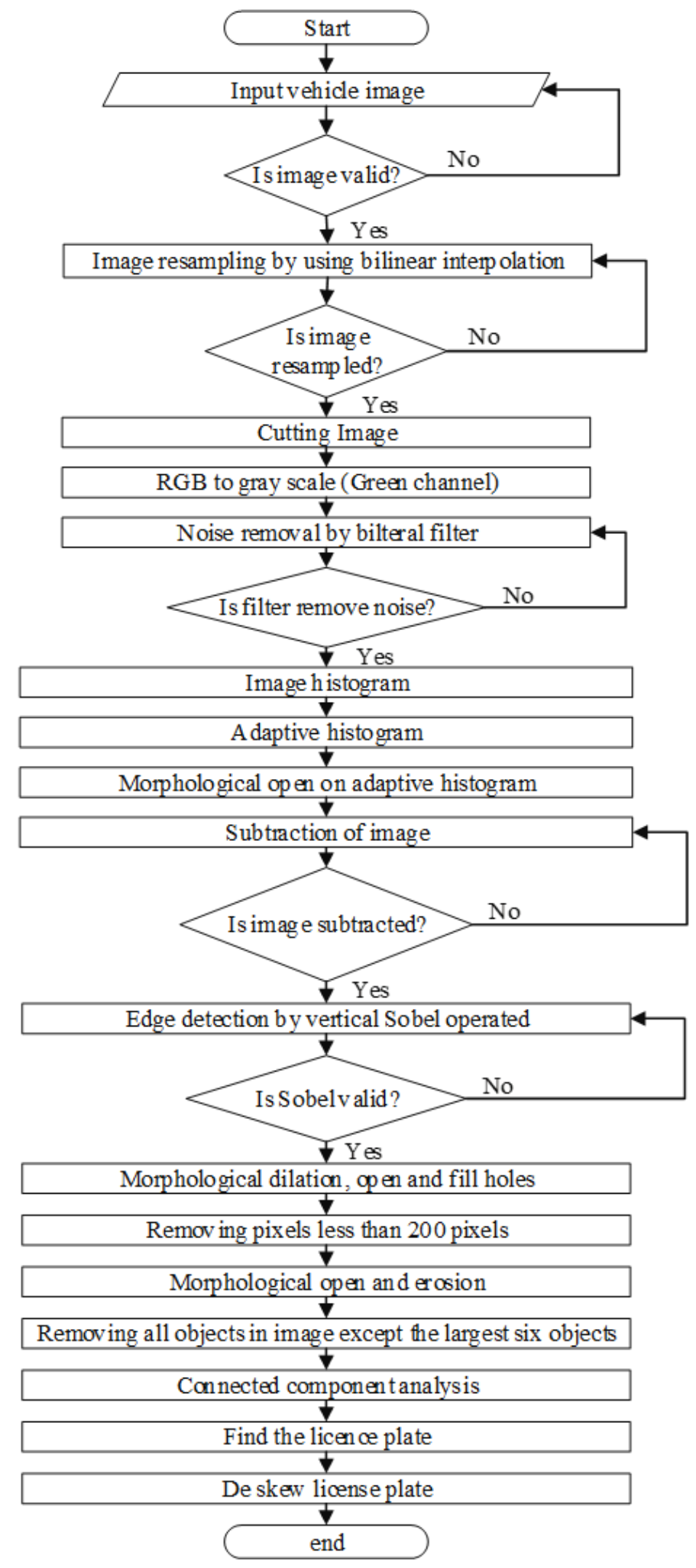

Figure 2. Flow chart of the proposed system 
For clarifying the working phases of the proposed method in detail, the steps are explained as follows.

\subsection{Preprocessing}

The preprocessing phase includes different steps that can be explained as follows.

\subsubsection{Image acquisition}

Firstly, the image of a vehicle is obtained by a digital camera with high resolution in various sizes, illumination conditions, and angles. Figure 3 illustrated an example.

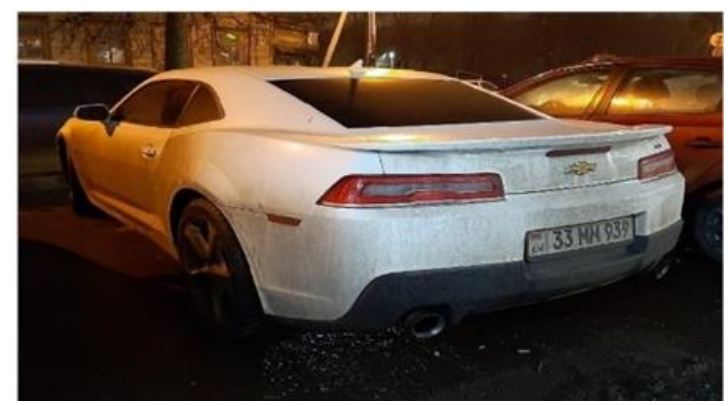

Figure 3. Original image

\subsubsection{Resampling and resizing images}

We must deal with the different dimensions of car images (Width $\mathrm{x}$ Height), where there are enormous numbers of pixels to be processed, and the run-time is increased. It is crucial to reduce the dimension and save the quality of the image. Image resampling technique (bilinear interpolation) is used to decrease the dimensions to (1024 x 768) pixels and keep the quality of the picture. It is used widely in the computer vision field to get pixel values for positions which it is not located in the pixel grid. Four neighbor values are adopted to compute the interpolated value in each sub-pixel [13]. Figure 4 stated the interpolation grid, while Figure 5 displays the resampling image. Then, after the image is resampled, it is resized to concentrate on the license plate region with dimensions of 940x 730, as shown in Figure 6.

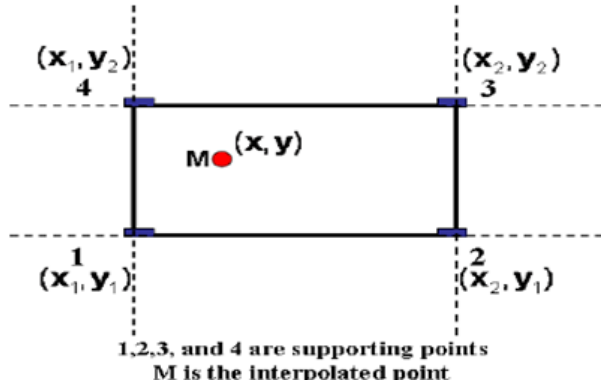

Figure 4. Interpolation grid

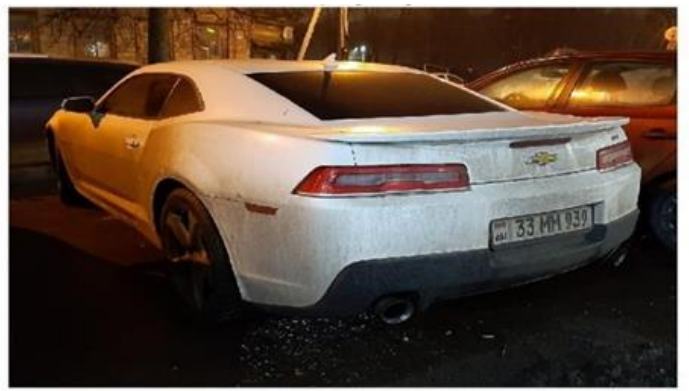

Figure 5. Resampling image

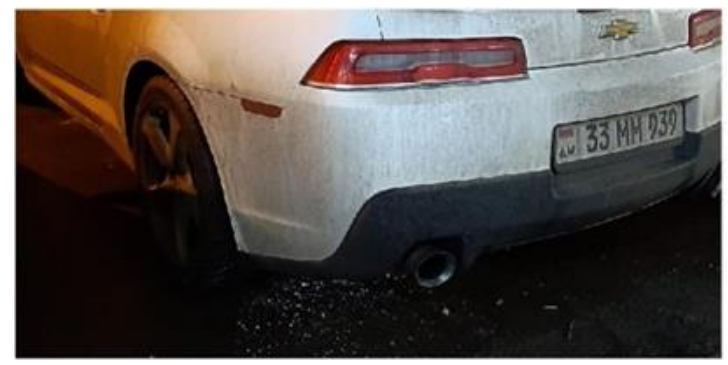

Figure 6. Resizing the resampling image 


\subsubsection{RGB to grayscale (green channel)}

The resized image in the previous step is RGB image contains three channels red, green, and blue. The value of each channel in the range 0-256, whereas the grayscale image contains just one channel. Thus, the extraction of one channel decreases the run time, as well as the storage space. We use the green channel because it gives adequate contrast for the image by experiments. Thus, we take the green channel as a proposed method for gray conversion $[14,15]$. Thus, the green channel is extracted, as apparent in Figure 7.

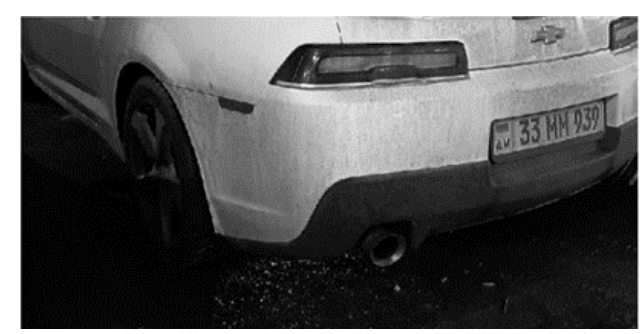

Figure 7. Green channel

\subsubsection{Bilateral filtering}

This filter smooths the car image while preserving its edges, through a non-linear mixture of adjacent values to an image. The way is non-iterative, straightforward, and local. It combines gray range or colors depend on both their geometric adjacency and their photometric hassling. It produces no illusion colors through edges in RGB-images [16]. Figure 8 explains this process.

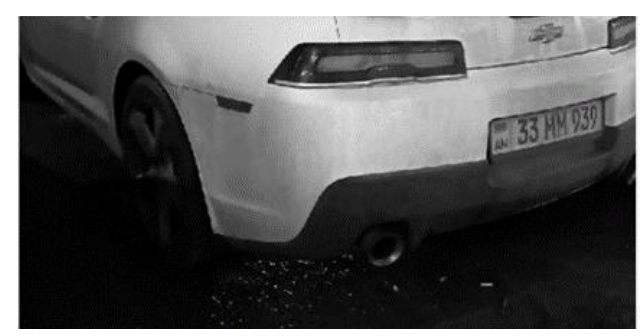

Figure 8. Bilateral filtering

\subsubsection{Image histogram and adaptive histogram}

The image histogram is a diagram in which the pixel's intensity ( $\mathrm{x}$-axis coordinate), which represents obtainable gray range against no. of the pixels ( $y$-axis coordinate) that refers to a specific pixels number to gray-range value. According to the adaptive- histogram, the total histogram of an image may have an extensive division, where local regions histogram is extremely skewed to once of the grey spectrum end. Therefore, we use adaptive histogram equalization in which processing differently for different areas [17]. Figure 9 clarifies the histogram of the image, while Figure 10 explains the adaptive histogram.

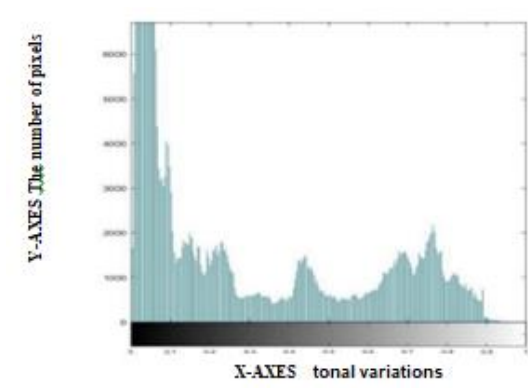

Figure 9. Image histogram

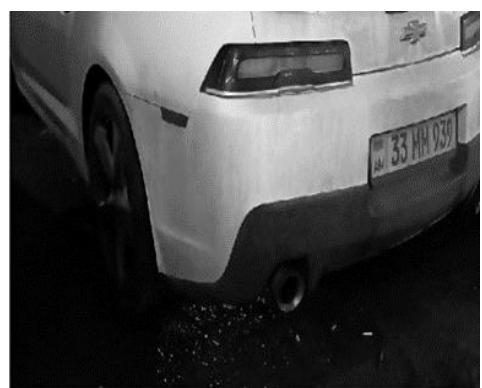

Figure 10. Adaptive histogram 


\subsection{License plate detection}

The detection of the plate is the important phase in the proposed method. The steps of the workflow of this phase are explained as follows.

\subsubsection{Morphological open and image subtraction}

Morphological operations are simple to use and based on set of theories. The objective of using morphological-operations is to remove the imperfections in the image structure. Morphological-open is applied to the (Adaptive-Histogram) images using a structuring element (disk), where the morphologicalopen performs erosion and then dilation operation. It smooths the object's outline. The morphological open is subtracted from the images of the Adaptive Histogram. Image subtraction is useful to remove the parts that are out dominated the images, such as a car body. This is to easy the localization of the plate in the next step. The image subtraction results are called the candidate images because the objects that remained from this step are areas that became a candidate location of a license plate [18, 19]. Figure 11 and Figure 12 identifies morphological-open and image-subtraction.

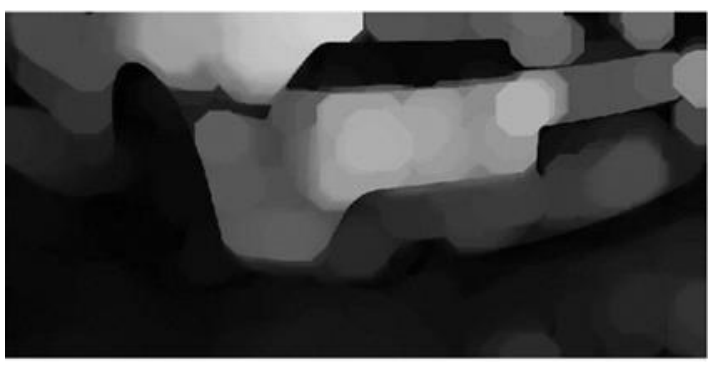

Figure 11. Morphological open

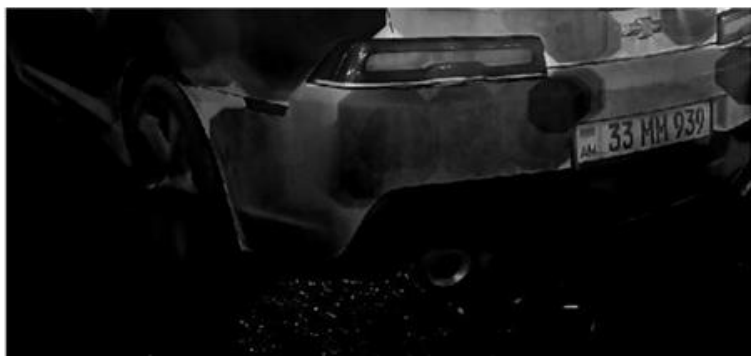

Figure 12. Image subtraction

\subsubsection{Sobel edge detection}

Vertical Sobel operator used to identify the edges in images. It generates two-dimensions spatial gradient measurement on the image and affirms higher spatial frequency regions that are corresponding to the edge [20]. The operator involves a twice $3 \times 3$ kernels, as illustrated in Figure 13.

\begin{tabular}{|l|l|l|}
\hline-1 & 0 & +1 \\
\hline-2 & 0 & +2 \\
\hline-1 & 0 & +1 \\
\hline
\end{tabular}

Gx

\begin{tabular}{|c|c|c|}
\hline+1 & +2 & +1 \\
\hline 0 & 0 & 0 \\
\hline-1 & -2 & -1 \\
\hline
\end{tabular}

Gy

Figure 13. Sobel convolution kernels

For getting distinct gradient component measurements in both orientations, Gx and Gy kernels can be applied to the image separately. The gradient magnitude and an approximate magnitude computed by the following equations [21]:

The magnitude $|G|=\sqrt{G x^{2}+G y^{2}}$

Approximate magnitude - $|G|=\left|G_{x}\right|+\left|G_{y}\right|$

After first order Sobel edge detection, Dilation operation shows visibility and the small's holes filled in objects. It is applied to Sobel edge detected images for more precise and comfortable to extract the License Plate. Figure 14 clarifies Sobel detection, while Figure 15 represents the dilation. 


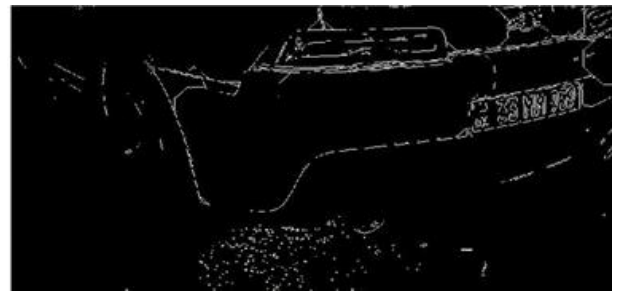

Figure 14. Sobel edge detection

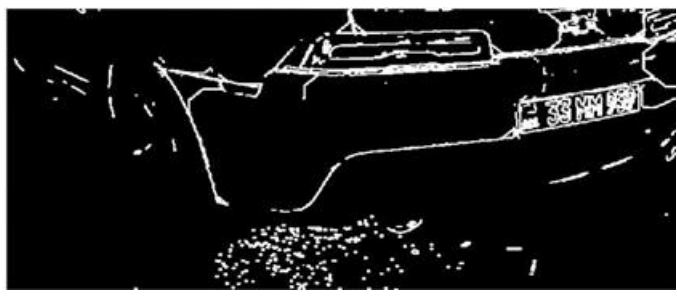

Figure 15. Morphological dilation

\subsubsection{Morphological open}

We apply another morphological-open on the dilated images, as shown in Figure 16.

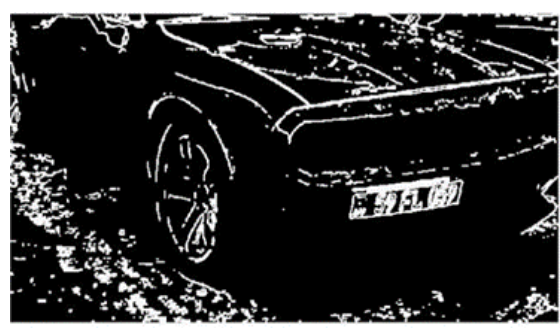

Figure 16. Morphological open

\subsubsection{Fill holes}

Filled Holes operation was used after the previous step, as shown in Figure 17, and then the proposed method removes objects less than 200 pixels, as shown in Figure 18. Then, morphological open is applied and passed to erosion, as shown in Figure 19 and Figure 20, respectively.

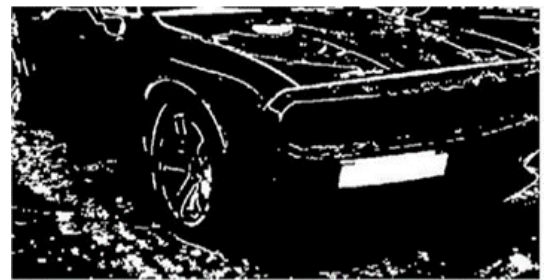

Figure 17. Filled holes

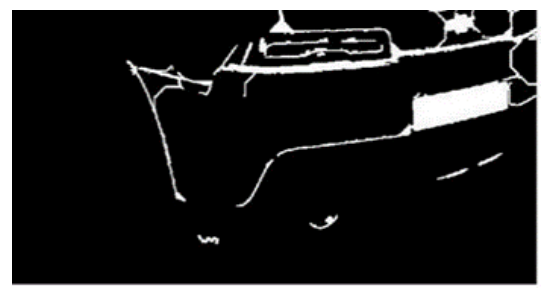

Figure 19. Morphological open

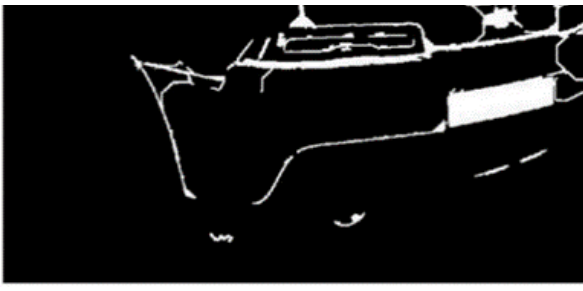

Figure 18. Removing pixels less than 200 pixels

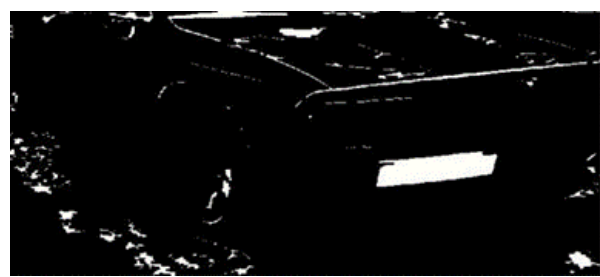

Figure 20. Morphological erosion

\subsubsection{Removing unwanted objects}

In this stage, only six-biggest objects are remained. After that, we used the connected component analysis to get the interested zone, which is the car plate, as apparent in Figure 21. 


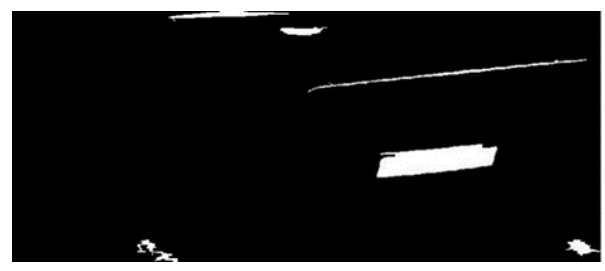

Figure 21. Removing unwanted objects

\subsubsection{Plate segmentation}

The connected component analysis is used for detecting the connected regions ( white parts ) in the binary-image. Connected Component labels are based on the given heuristic [22]. Then, the adopted conditions to extract a plate, which are the size and dimensions of the plate area, filling area, and the aspect ratio (Height/Width), to find the accurate license plate. Then, the proposed method cuts this area from the adaptive histogram image according to those dimensions, as shown in Figure 22.

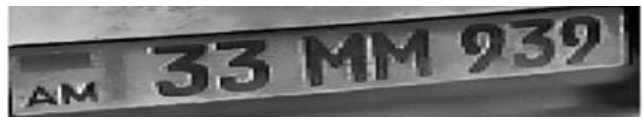

Figure 22. License plate

\subsubsection{Rotate the skew plate}

The previous result of a segmented plate can be a skew plate, so we use the Hough transform for line detection and bilinear interpolation to rotate the plate. We use the canny edge detector to discover edges, as shown in Figure 23.

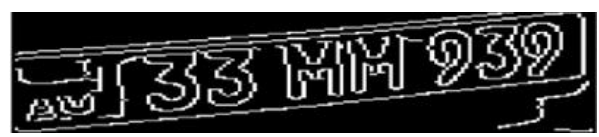

Figure 23. Canny edge detector

For line detection, we used the Hough transform, which is the most famous technique for the detection of the straight line in a digital image, where it is a linear transform. Hough space uses two parametric for detection of a line in the image and represented as follows [23, 24]:

Where $L$ is a straightforward-line in the space of the image. The variable $\theta$ is the angle to the straight-line that crosses through the origin being orthogonal to the straight line $L$. The variable $\rho$ considered the area from the origin to the line $L$. The straight- line is available to use the polar coordinate system as shown in Figure 24.

$$
p=x \cos \theta+y \sin \theta
$$

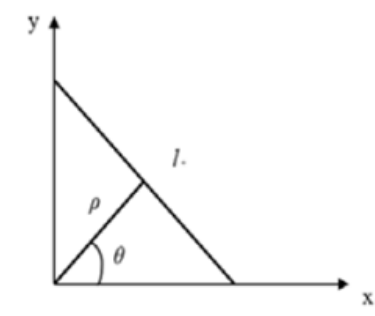

(a)

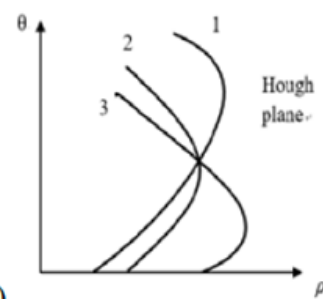

(b)

Figure 24. Hough transform: (a) the Cartesian coordinate system; (b) the polar coordinate system 
The peaks are identified by the Hough Space Accumulator. Hough Space accumulator is used to locate the regions in which most Hough space lines are intersected at a common point in the $(\rho, \theta)$ as illustrated in Figure 25. Then, the proposed method finds the longest-line segment, as shown in Figure 26 [25-27]. After determining the most extended line angle, we rotate the plate using the Bilinear interpolation technique, which is used to deskew the car plate without losing the quality of the image[28]. Figure 27 displays the rotation of the plate using Bilinear interpolation.

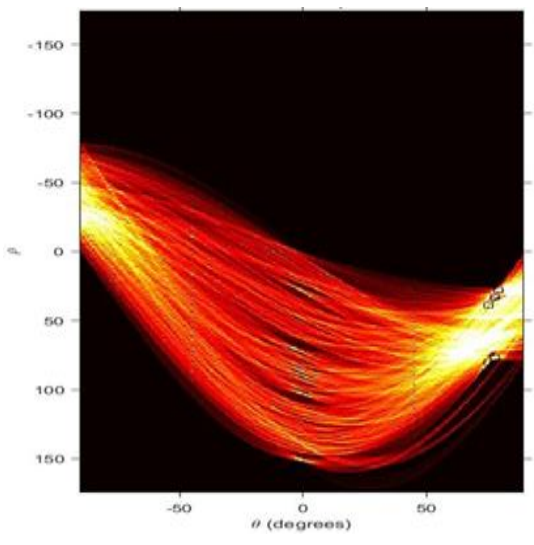

Figure 25. Accumulator space

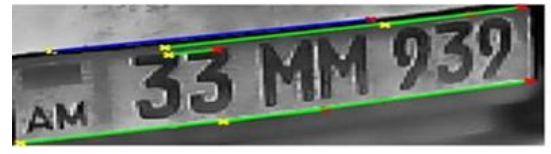

Figure 26. The most extended- line segment (the line with blue color)

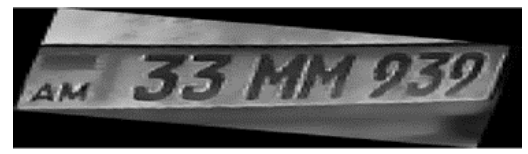

Figure 27. Deskew license plate

\section{RESULTS AND DISCUSSION}

In order to test the proposed method, four groups of vehicle plates are considered. These groups are collected from four countries, which are Iraq, Armenia, Hungarian, and Belarus, with a total number of 860 images with different illumination conditions and orientations.

MATLAB 2019a simulator is adopted in this paper to represent the proposed method as well as testing it with numerous case studies. The reason behind adopting this simulator is the availability of software components regarding image processing.

Table 1 shown the result obtained of the proposed method. It can be seen that the suggested method shows an excellent performance in terms of computed accuracy (extraction percentage). The diversity of accuracy percentage between the adopted image groups is the result of the resolution of them as well as the angle of the image capturing in addition to noise and surrounding circumstances. It is essential to note that the proposed method works well even under difficult conditions of import images with little contrast, noisy and low-resolution in addition to various distances.

Table 1. The proposed method performance

\begin{tabular}{|c|c|c|c|c|c|c|c|}
\hline ID & $\begin{array}{l}\text { The type of } \\
\text { license plate }\end{array}$ & $\begin{array}{c}\text { Total } \\
\text { images }\end{array}$ & $\begin{array}{l}\text { No. of extracted } \\
\text { license plate }\end{array}$ & $\begin{array}{l}\text { No. of not extracted } \\
\text { license plate }\end{array}$ & $\begin{array}{c}\text { The percentage of } \\
\text { deskew plate } \%\end{array}$ & $\begin{array}{l}\text { Run time } \\
\text { in Sec. }\end{array}$ & $\begin{array}{c}\text { License plate } \\
\text { Extraction percentage }\end{array}$ \\
\hline 1 & Iraqi plate & 104 & 102 & 2 & $100 \%$ & 8 & $102 / 104=98.07 \%$ \\
\hline 2 & Armenia plate & 333 & 331 & 2 & $100 \%$ & 8 & $331 / 333=99.39 \%$ \\
\hline 3 & Hungarian plate & 188 & 186 & 2 & $100 \%$ & 8 & $186 / 188=98.93 \%$ \\
\hline 4 & Belarus plate & 235 & 234 & 1 & $100 \%$ & 8 & $234 / 235=99.57 \%$ \\
\hline Total & & 860 & 853 & 7 & $100 \%$ & & $98.99 \%$ \\
\hline
\end{tabular}

\section{CONCLUSION}

We proposed an efficacious approach for the vehicle plate extraction using Bilateral filter and Hough transform. It was based on the presented algorithm of [12] that has been modified. The modification was focused on the resizing of images without affecting the resolution. The proposed method consisted of three stages: preprocessing, license plate detection, and Deskew plate. The proposed method was tested with four different multinationals car images from the following countries: Iraq, Armenia, Belarus, Hungary. It had been proved as an efficient method in performing the plate extraction and localization. It is efficient with different weather conditions, such as sunny, mud, cloudy, night, rainy, and different orientations. The accuracy for extraction and rotation plates is $98.99 \%$ and $100 \%$ for rotation images. 


\section{REFERENCES}

[1] X. A. Davix, L. Oshin, and P. Shamili, "License plate localization by sobel vertical edge detection method," Int. J. Emerg. Technol. Eng. Res., vol. 4, no. 6, pp. 48-53, 2016.

[2] O. Khin, M. Phothisonothai, and S. Choomchuay, "License plate detection of Myanmar vehicle images captured from the dissimilar environmental conditions," in 2017 International Conference on Advanced Computing and Applications (ACOMP), pp. 127-132, 2017.

[3] G. Rabbani, M. A. Islam, M. A. Azim, M. K. Islam, and M. M. Rahman, "Bangladeshi license plate detection and recognition with morphological operation and convolution neural network," in 2018 21st International Conference of Computer and Information Technology (ICCIT), pp. 1-5, 2018.

[4] X. A. Davix, C. S. Christopher, and S. S. Christine, "License plate detection using channel scale space and color based detection method," in 2017 IEEE international conference on circuits and systems (ICCS), pp. 82-86, 2017.

[5] M. R. Asif, Q. Chun, S. Hussain, M. S. Fareed, and S. Khan, "Multinational vehicle license plate detection in complex backgrounds," J. Vis. Commun. Image Represent., vol. 46, pp. 176-186, 2017.

[6] M. S. Farag, M. M. M. El Din, and H. A. El Shenbary, "Parking entrance control using license plate detection and recognition," Indonesian Journal of Electrical Engineering and Computer Science (IJEECS), vol. 15, no. 1, pp. 476-483, 2019.

[7] I. Astawa, I. G. N. B. Caturbawa, I. M. Sajayasa, and I. M. A. D. S. Atmaja, "Detection of license plate using sliding window, histogram of oriented gradient, and support vector machines method," in Journal of Physics: Conference Series, vol. 953, no. 1, p. 12062, 2018.

[8] A. M. Ahmed and S. F. Aly, "Egyptian license plates recognition system using morphologial operations and multi layered perceptron," in ICT in our lives-2019, 2019.

[9] E. Rashedi and H. Nezamabadi-Pour, "A hierarchical algorithm for vehicle license plate localization," Multimed. Tools Appl., vol. 77, no. 2, pp. 2771-2790, 2018.

[10] P. Venkateswari, E. J. Steffy, and D. N. Muthukumaran, "License plate cognizance by Ocular character perception'," Int. Res. J. Eng. Technol., vol. 5, no. 2, pp. 536-542, 2018.

[11] S. S. Omran and J. A. Jarallah, "Automatic Iraqi cars number plates extraction," Iraqi J. Comput. Informatics ijci, vol. 44, no. 1, pp. 34-41, 2018.

[12] S. Kaur and S. Kaur, "An efficient method of number plate extraction from indian vehicles image," International Journal of Computer Applications, vol. 88, no. 4. pp. 14-19, 2014.

[13] S. A. Fahmy, "Generalised parallel bilinear interpolation architecture for vision systems," in Proceedings - 2008 International Conference on Reconfigurable Computing and FPGAs, ReConFig 2008, pp. 331-336, 2008.

[14] M. M. A. O. Bagabir, "Sudanese vehicles license plate recognition," PhD Thesis. Sudan University of Science and Technology, 2016.

[15] M. AbdulraheemFadhel, A. J. Humaidi, and S. Razzaq Oleiwi, "Image processing-based diagnosis of sickle cell anemia in erythrocytes," in 2017 Annual Conference on New Trends in Information \& Communications Technology Applications (NTICT), pp. 203-207, 2017.

[16] C. Tomasi and R. Manduchi, "Bilateral filtering for gray and color images," in Sixth international conference on computer vision (IEEE Cat. No. 98CH36271), pp. 839-846, 1998.

[17] A. Vyas, S. Yu, and J. Paik, "Fundamentals of digital image processing," Signals and Communication Technology. pp. 3-11, 2018,

[18] D. Avianto, "Indonesian license plate extraction using morphological opening operatiom," Int. J. Sci. Eng. Technol. $U T Y$, vol. 1, no. 1, pp. 44-52, 2018.

[19] H. Al-Yassin, J. I. Mousa, M. A. Fadhel, O. Al-Shamma, and L. Alzubaidi, "Statistical accuracy analysis of different detecting algorithms for surveillance system in smart city," Indonesian Journal of Electrical Engineering and Computer Science (IJEECS), vol. 18, no. 2, pp. 979-986, 2020.

[20] D. Biswas, P. De, and D. Bhattacharya, "Fire Disaster alert system using sobel edge detection," Available SSRN 3512026, 2019.

[21] S. Gupta and S. G. Mazumdar, "Sobel edge detection algorithm," Int. J. Comput. Sci. Manag. Res., vol. 2, no. 2, pp. 1578-1583, 2013.

[22] S. S. G. Mercy, I. Muthulakshmi, and P. G. Scholar, "Automatic number plate recognition using connected component analysis algorithm," Int. J. Technol. Res. Eng., vol. 5, no. 7, 2018.

[23] P. Li, M. Nguyen, and W. Q. Yan, "Rotation correction for license plate recognition," in 2018 4th International Conference on Control, Automation and Robotics (ICCAR), pp. 400-404, 2018.

[24] L. Alzubaidi, M. A. Fadhel, O. Al-Shamma, and J. Zhang, "Robust and efficient approach to diagnose sickle cell anemia in blood," in International Conference on Intelligent Systems Design and Applications, pp. 560-570, 2018.

[25] P. S. Rahmdel, R. Comley, D. Shi, and S. McElduff, "A review of hough transform and line segment detection approaches.," in Proceedings of the 10th International Conference on Computer Vision Theory and Applications, vol. 2, pp. 411-418, 2015.

[26] M. H. Dashtban, Z. Dashtban, and H. Bevrani, "A novel approach for vehicle license plate localization and recognition,” Int. J. Comput. Appl., vol. 26, no. 11, pp. 22-30, 2011.

[27] N. S. Aminuddin, "A new approach to highway lane detection by using Hough transform technique," J. Inf. Commun. Technol., vol. 16, no. 2, pp. 244-260, 2020.

[28] B. M. Nema and E. A. Mohammad, "Real time recognition and tracking of iraqi vehicle license plate," Int. J. Digit. Content Technol. its Appl., vol. 10, no. 2, pp. 25-32, 2016. 